\title{
II Reunión General del Grupo Latinoamericano de Instituciones del Cemento y del Concreto
}

\author{
Río de Janeiro, Brasil, 15-17 de octubre 1979
}

\section{Consideraciones sobre la economía de combustibles y otros derivados del petróleo en la fabricación y en la utilización de los cementos}

Prof. Dr. J. CAlleJ. Vicedirector del IETCC

MADRID (España)

\section{E X O R D I O}

A la vista del Programa de la II Reunión General del Grupo Latinoamericano de Instituciones del Cemento y del Concreto, el que escribe no ha podido - ni querido- resistir la tentación de hilvanar unas observaciones y comentarios de propia cosecha, acerca de puntos que, estando previstos aisladamente en el temario, como son, entre otros, la normalización de los cementos, la promoción directa e indirecta del empleo del hormigón, la investigación científica y tecnológica en el campo de ambos materiales, la automatización de los procesos de fabricación del cemento, etc., tan íntimamente ligados pueden estar -y de hecho lo están- entre sí y con el que parece ser -y en todo caso podría ser por su interés y actualidad - el tema central del Programa: La Economía de Combustibles derivados del Petróleo, que no en vano figura en cabeza del mismo.

El que esto escribe no ha tenido conocimiento previo de lo que aquí se va a tratar acerca de los mencionados puntos y tema central. Y, sin embargo, tiene la osadía de arriesgarse a tratar de relacionar unos y otro, tal vez de una forma superficial, eso sí, pero a su modo de ver directa y evidente.

Quiérase ver en ello, no la osadía, que no es del caso, sino el buen deseo de cooperar con una modestísima aportación española a esta II Reunión, por lo poco - si algo- que dicha aportación pueda tener de interés para los reunidos. 
Por ello, naturalmente, se va a exponer en lo que sigue la situación y postura actual de España frente a algunos aspectos del tema, siempre y solamente desde el punto de vista de quien les habla, a título personal y sin connotación alguna de cargo o representación.

En cualquier caso, gracias del autor por su venia, en el doble sentido de perdón por su atrevimiento y de licencia para dirigirse a ustedes.

\section{A T E R I A}

Dentro del tema general del ahorro de energía a escala mundial, en cualesquiera usos y consumos y en no importa qué procesos industriales, es sabido que la Agencia Internacional de la Energía ha dedicado una parcela de su atención al ahorro de combustible (fuel-oil) en la fabricación de cemento en el mundo.

Ello ha sucedido así como consecuencia de la crisis energética general surgida a raiz de 1973, y agravada de forma acelerada en los años siguientes, sin solución de continuidad hasta nuestros días.

Las repercusiones que la proyección de esta crisis ha tenido y puede aún tener en el ámbito mundial de la fabricación del cemento son muchas y grandes. Y, además, muy variadas.

Precisamente ya en la I Reunión General de este Grupo, celebrada en Medellín (Colombia) y diciembre de 1973, uno de los dos representantes estadounidenses del ACI asistentes a la misma, el Dr. PHILLEO, señaló que, entre las tres principales razones de la ya entonces seria escasez de cemento en los Estados Unidos figuraba, tal vez en cabeza, la penuria de combustible, y concretamente de gas natural, para la industria cementera norteamericana. Y añadió que, entre los tres posibles remedios para paliar tal penuria y, en consecuencia, tal escasez de cemento, se contaba, quizás en primer lugar, con la regresión al empleo del carbón como fuente de combustible.

Hay que destacar el valor de esta expresión, porque se habló del carbón, no como combustible, sino como fuente de combustible. $\mathrm{Y}$ hay que poner en ello mayor énfasis, si cabe, por haber sido dicho por un destacado especialista de un país que, como los Estados Unidos de Norteamérica, posee abundantes yacimientos de carbón de excelente calidad. Tal vez quiso referirse el Dr. PHILLEO, siquiera no más que de pasada, dada la ocasión, a la hidrogenación y a la gasificación del carbón, ya que de la hulla se pueden extraer, aunque con mayor trabajo y costo, productos del mismo valor energético que los del petróleo.

A mayor abundamiento, hay que meditar sobre el hecho de que las reservas mundiales de petróleo se han calculado para 30-40 años -y de esto ya hace algunos-, y de que su sustitución gradual por recursos nucleares se va produciendo con retraso; mientras que las reservas de carbón en el mundo están calculadas para un consumo de mil a mil quinientos años, según zonas del planeta.

Pero, ¿qué decir al respecto en una situación como la de España, país con carbón muy escaso y mal repartido, y por añadidura de muy baja calidad? No ha de olvidar quien lo sepa, y ha de saberlo quien lo ignore, que en el decenio de los 40 y aún después, se quemaban en los hornos de las fábricas españolas de cemento carbones con contenidos de 
ceniza idel 50 por ciento y aún mayores!, con una amplia variabilidad de materias volátiles y, por supuesto, de potencia calorífica, en todo caso baja.

Hasta el punto de que para un director técnico de fábrica era entonces más preocupante la gran fluctuación de la calidad del carbón que la baja condición de la misma: "denme carbones uniformemente malos, antes que variablemente menos malos", solían decir, porque de carbones regulares o buenos no se podía hablar.

$\mathrm{Y}$ hay que añadir que al bajo rendımiento térmico de los carbones, y como consecuencia del mismo, había que sumar la formación de anillos en los hornos, el quebranto del refractario, y las paradas de marcha, con la consiguiente merma de producción y de calidad, y el mayor costo del proceso $y$ del producto.

Y cabe preguntarse si tales carbones ineptos para ser quemados van a ser suficientemente aptos para ser hidrogenados y gasificados. La respuesta apriorística parece ser negativa, con lo cual tal solución no parece ser en principio la más idónea, al menos para el caso español.

La drástica reducción del suministro de gas natural a las fábricas de cemento de los Estados Unidos en 1973, unida al recrudecimiento de las normas anticontaminación y a la falta de rentabilidad del cemento por fijación y congelación de su precio, hicieron que este país recurriera en parte a la importación. Y lo hizo antes que recurrir a ninguna de las tres soluciones apuntadas entonces por el Dr. PHILLEO, a saber: el ya citado regreso al empleo del carbón - como tal a gasificado-; la atenuación de las exigencias contra la contaminación mientras durase la crisis de la energía (hoy se ve que este remedio no se hubiera podido mantener, so pena de renunciar definitivamente a las medidas anticontaminantes, pues la crisis continúa corregida y aumentada y "en vías de mayor desarrollo"); y la liberalización del precio del cemento.

Sólo así pudieron los Estados Unidos, a partir de entonces, ir enjugando el déficit de producción y de demanda cifrado en 6 millones de toneladas en 1973, y previsto en 12 a 38 millones para 1980, según distintas estimaciones hechas a la sazón.

Una vez más no es ésta la solución para España, país hoy día, y con mucho, a la cabeza de los exportadores mundiales de clínker y cemento -incluso a los propios Estados Unidos-, amén de ser el cuarto productor de Europa y el séptimo mundial.

Todo lo más a que puede aspirar España, dentro del capítulo del consumo energético para la fabricación de cemento es, o bien a la búsqueda, hallazgo y explotación de recursos propios, o bien a la importación de gas natural africano de Argelia y Túnez. Ambas soluciones a largo plazo, sobre todo la primera de ellas, están en marcha.

Como solución más inmediata, y ya puesta en ejecución en España, está la de fabricar y utilizar cementos con adiciones o, como llaman los franceses. "cementos con constituyentes secundarios", o "blended cements", al decir anglosajón.

Las adiciones o constituyentes secundarios pueden ser de naturaleza muy diversa, desde las hidráulicamente activas "per se", como las escorias siderúrgicas (básicas granuladas de horno alto), o las potencialmente activas frente al clínker, como las puzolanas naturales, o artificiales - cenizas volantes y arcillas térmicamente activadas-, hasta incluso las químicamente inertes ("fillers" calizos, principalmente), aunque físicamente operantes.

Esta solución, por razones técnicas de utilización de los cementos muy anteriores a la crisis energética de los combustibles (y tales como la estabilidad y la durabilidad o re- 
sistencia química de los hormigones), se ha adoptado a escala mundial en general, y europea en particular, desde hace mucho tiempo, como es bien sabido.

El principio no puede ser más sencillo: si el consumo calorífico en la fabricación es de $950 \mathrm{kcal} / \mathrm{kg}$ de clínker producido, y la potencia calorífica del fuel-oil es de $9.500 \mathrm{kcal} / \mathrm{kg}$ de fuel quemado, se gastan $100 \mathrm{~kg}$ de fuel-oil para producir una tonelada de clínker. Por lo tanto, sustituciones entre, por ejemplo, 20 por ciєnto de clínker (cementos españoles PA) y 80 por ciento de clínker (cementos españoles S-III) por adiciones adecuadas, suponen ahorros de fuel-oil de iguales cuantías, es decir, entre 20 y $80 \mathrm{~kg}$ de fuel-oil por tonelada de clínker producida.

Verdad es que la extracción de las adiciones y/o su tratamiento y transporte pueden consumir combustible, incluso carburante derivado del petróleo, probablemente más caro que el propio fuel-oil que se quema en un horno de cemento. Por lo tanto, es preciso hacer dos observaciones: una, que los ahorros indicados son ideales, teóricos o máximos; otra, que para que la adición utilizada en la proporción añadida sea rentable, esto es, para que el ahorro real sea sustancioso, es preciso que dichos gastos energéticos de extracción, tratamiento y transporte sean aceptables, es decir, no muy elevados. Lo que a su vez implica una distancia máxima de transporte de las adiciones desde su fuente de origen hasta la planta en que se utilizan. Y también, que la proporción de adición sea real y económicamente remuneradora.

En este aspecto hay que añadir que las adiciones - "fillers" - de tipo calizo, disponibles siempre en cantidad en cualquier fábrica de semento, no consumen fuel-oil en tratamientos, y que sus gastos de extracción y manipulación en una fábrica son prácticamente los mismos que le corresponden de todos modos como materia prima del crudo, pero sin pasar por el horno.

En cuanto a las escorias y cenizas volantes, si quedan como materiales de desecho en escombreras, a la postre hay que eliminarlas : lo cual exige manipulación, transporte y gasto energético, pero sin el beneficio de sus energías potenciales que quedan así desperdiciadas.

Es cierto, por otra parte, que las escorias utilizables para cemento requieren un enfriamiento - templado en aire o en agua - más rápido e intenso que el ordinario, lo cual supone un gasto adicional.

Si se considera un espectro energético más amplio que el del horno, dentro de una fábrica de cemento, el consumo de $k$ Wh en molienda de cemento se puede cifrar alrededor - tal vez en algo más- de $100 \mathrm{kWh} / t$ para portland ordinario a base de clínker y yeso. Este consumo puede variar en un sentido u otro, según la naturaleza y proporción de las adiciones sustituyentes del clínker.

De acuerdo con su naturaleza, porque las adiciones más duras que el clínker consumirán más que éste, y las menos duras consumirán menos, para una misma finura del cemento resultante. Así, las puzolanas naturales suelen ser por lo general algo más blandas que el clínker, mientras que algunas escorias suelen ser algo más iduras. Las cenizas volantes, en cambio, son de por sí muy finas por su origen y formación.

Pero la cuestión global es más compleja, por cuanto que los cementos con adiciones en cuantías considerables - los puzolánicos con 25-35 por ciento de puzolanas y los siderúrgi$\cos$ y de horno alto ("cementos de escorias") con 50-80 por ciento de escorias- se suelen moler a mayor finura que los portland sin adición, por ser cementos "más lentos" que éstos, sobre todo para una finura igual. 
En definitiva, lo que podría ahorrarse en un semento puzolánico en razón de utilizar una puzolana más blanda que el clínker habría que gastarlo en obtener un cemento de mayor finura que el portland sin adición, por la razón expuesta.

Pero, por otra parte, la caliza o marga que como materia prima del crudo correspondería a la parte de clínker sustituida por la adición, tendría que sufrir dos procesos de molienda -aparte de los de trituración en cantera-: uno, como "crudo", antes de pasar por el horno; otro, como "clínker", después de salir de éste. En cambio, la adición sólo pasa por el segundo proceso de molienda del cemento, lo cual i es otro factor positivo de ahorro de kilowatios.

En cualquier caso, el ahorro principal de energía que lleva aparejada la fabricación de cementos con adiciones estriba en la cantidad de material que deja de pasar por el horno; es decir, se trata, en principio, de un ahorro de calorías, más que de kilowatios, y por lo tanto de una economía de fuel-oil. Esto es importante, porque los recursos energéticos conflictivos y causantes de la crisis energética en general, son el petróleo y sus derivados, en particular.

En este sentido cabe hacer algunas consideraciones para salir al paso de posibles tergiversaciones al respecto. Se ha indicado antes que los cementos que contienen puzolanas (incluidas como tales las cenizas volantes) o escorias son más lentos que los portland sin adiciones, es decir, que desarrollan sus resistencias más despacio, por el simple hecho de que las adiciones que contienen son, respectivamente, inactivas (prácticamente inertes), o menos activas a edades cortas, sobre todo si estos cementos proceden del mismo clínker y/o tienen la misma finura que el cemento portland "puro" de referencia.

Por lo tanto, en estas circunstancias, entre un cemento portland sin adiciones y otro con ellas, siendo ambos de la misma categoría resistente, esto es, cumpliendo ambos con los requisitos de resistencias mínimas correspondientes a dicha categoría, el primero (sin adiciones) dará a todas las edades, y sobre todo a las más cortas, resistencias mayores que el segundo (con adiciones).

Esto quiere decir que, en igualdad de condiciones, el hormigón hecho con el segundo cemento endurece más lentamente que el hecho con el primero o, lo que es lo mismo, que para alcanzar la misma resistencia que éste, aquél necesita más tiempo, lo cual exige en su caso demorar un tanto más su desencofrado. En resumidas cuentas, retrasar la obra.

Ahora bien, no se puede olvidar que lo que se pretende en este caso con los cementos que contienen adiciones es economizar fuel-oil, que es el recurso del que se puede llegar a escasear o carecer, y eso no resulta - no puede de ningún modo resultar - gratuito, sino que hay que pagarlo en una moneda que - precisamente en este caso- es un mayor tiempo de encofrado.

De este tiempo se dispone siempre con amplio margen, aunque resulten más caras las obras, lo cual es otra forma de pagar (conseguir) el menor consumo de una materia como el fuel-oil, que es (y va a serlo más) escasa, difícil de adquirir y cara.

No es válido el "argumento" de que el ahorro de fuel-oil es ficticio porque para conseguir en obra la misma resistencia en igual plazo y desencofrar en el mismo tiempo habría que utilizar dosificaciones mayores en el caso de hormigones de cementos con adiciones que en el caso de los mismos hormigones de cemento sin ellas, con lo cual la economía de combustible desaparecería. 
Porque no se trata de eso. Hay que repetir una vez más que se trata de pagar el ahorro de algo que escasea, es caro y hay que abonarlo la mayor parte de las veces - hablo de España- en divisas, con algo de lo que se dispone en abundancia y que, si bien no es barato, se puede pagar en moneda nacional: la mano de obra.

El hecho de que la peor parte de este "negocio" le corresponda, por ahora, al constructor y no al fabricante de cemento es una mera casualidad debida a la propia naturaleza de los hechos y de las circunstancias. $\mathrm{Y}$ de no aceptar éstos tal y como son, no hay reducción del consumo de fuel-oil en fabricación de cemento que valga.

Porque, en efecto, en maquinaria y en proceso, en cuanto a aprovechamiento y recuperación de calor se refiere, con los más modernos equipos y sistemas se ha alcanzado o se está a punto de alcanzar prácticamente un límite mínimo de consumo térmico en la fabricación del clínker portland clásico y tradicional. En este concepto se puede considerar incluida la automatización, aún la más avanzada.

La automatización facilita, regulariza y controla el proceso y, si se quiere, también la calidad del cemento. Reduce mano de obra de unos determinados niveles y exige técni$\cos$ o especialistas a otros niveles más altos. Puede, incluso, rebajar el costo total del proceso y del producto; pero es dudoso que, dentro de ello, pueda reducir, significativamente, el consumo de combustible en el horno.

Porque si la automatización regulariza el proceso y la calidad y rebaja el costo, exige a cambio condiciones a las materias primas y a los combustibles. $\mathrm{Y}$, al tenerse que ajustar a un "modelo" que impone un estrecho margen de variabilidad a los parámetros que lo definen, no puede admitir desequilibrios fuertes de dichos parámetros y, en consecuencia, no es operante en procesos que utilicen como combustible carbones de baja calidad fuertemente fluctuantes.

Por otra parte, el desarrollo de posibles nuevos tipos de cemento y de posibles tipos nuevos de proceso, basados tanto en la termodinámica del horno como en la fisicoquímica del clínker y de la clinkerización, van a exigir tal vez un tiempo del que no se dispone, para resolver la presente situación energética, cada vez más agobiante, pues son soluciones a largo plazo - tal vez a muy largo plazo-; probablemente del máximo interés futuro, pero aún en etapa de incipiente investigación a escala de laboratorio.

Es en este terreno en el que la investigación científica (básica) y técnica (aplicada y de desarrollo) tanto tiene aún que hacer y puede hacer, a pesar de algunas apreciaciones en contrario. El estudio de nuevos sistemas de fases del clínker con nuevos eutécticos de más bajo punto de fusión; el empleo de fundentes y mineralizadores para rebajar sustancialmente la temperatura y/o el tiempo de clinkerización, y tantas otras posibilidades, son temas sugestivos para el investigador y de interés para el técnico.

Es éticamente obligado el abordarlos, si bien ha de hacerse con la mira bien fija en lo que se pretende conseguir, esto es: un material de construcción -el cemento- que, siendo posiblemente más barato, se pueda obtener con las materias primas clásicas y usuales, y mediante procesos y con equipos no muy diferentes de los tradicionales; y que tenga todas las buenas cualidades - y si es posible alguna más- de los cementos del presente, con menos o ninguno de sus inconvenientes.

Si esto se consigue, ello es, por añadidura, a base de crear nuevo conocimiento científico y técnico, lo cual supone el logro de un objetivo doble. Porque si la orientación inicial es exclusivamente hacia el diletantismo, tal vez se logre conocimiento, pero éste será probablemente un conocimiento estéril. 
Se quiere decir con esto que la investigación en este campo ha de ser fundamentalmente aplicada, aún cuando tenga que echar mano, y pueda y deba hacerlo, de cuantos conocimientos y exploraciones en el terreno de la ciencia básica sean necesarios.

Se ha indicado anteriormente que la peor parte del "negocio" del ahorro de combustible en la fabricación del cemento afecta al constructor, "por ahora". Y se ha dicho así, porque bien pudiera ser que, allí donde se pueda - y según experiencia española pasada incluso allí donde no se pueda - haya que volver al carbón (bueno o malo), con lo cual, por mor de nuevos hechos y de otras circustancias, la peor parte correspondería entonces a los fabricantes de cemento.

En efecto, todas las dificultades de formación de anillos, paradas, mayor desgaste de refractario, etc., antes señaladas, encarecerían el proceso y el producto, y más si en tales condiciones se exige el mantenimiento en éste de una determinada calidad, a un mismo precio.

Pero mantener la misma calidad actual en tales circunstancias podría resultar, además de más caro, técnicamente imposible, con lo cual el quebranto del fabricante de cemento no beneficiaría en nada al constructor; antes bien, perjudicaría también a éste. El daño sería para ambos, y mayor para el país de ambos.

Dentro incluso de la posibilidad de mantener la calidad del cemento a mayor precio, el constructor sufriría también las correspondjentes consecuencias.

En definitiva, la solución de los cementos con adiciones se presenta como alternativa mejor — $\longrightarrow$ si se prefiere menos mala - para lograr ahorros de fuel-oil en fabricación de cemento. Así lo entiende la Agencia Internacional de la Energía.

Por supuesto, que esta solución exige estudio y experimentación previos. Antes de lanzar al mercado un nuevo cemento con adiciones es preciso conocer a fondo éstas y los clínkeres con los que se mezclan, así como fijar las proporciones óptimas o máximas admisibles en cada caso, y ensayar en hormigones reales los cementos que resulten.

Es necesario también fijar atinadamente las especificaciones para dichos cementos dentro de lasi normas, y ejercer el obligado control de los mismos, tanto en fábrica como en obra. Recomendable podría ser, a efectos de nivel y de regularidad de calidad, el establecimiento de autocontroles por parte de los fabricantes - aparte y como complemento auxiliar de otros controles, o en lugar de ellos-, así como de sellos, marcas o distintivos de calidad, para los diferentes tipos, clases y categorías de los cementos.

Todo esto es tanto más recomendable cuanto que se suele hablar de clínker y de adiciones como si uno y otras fuesen especies químicas puras, únicas e invariables, siendo así que en la realidad es todo lo contrario. Además, los mismos cementos de un determinado tipo y clase y categoría resistente pueden ser más diferentes entre sí que los de tipos, clases y categorías distintos.

Por ejemplo, un cemento con una resistencia de $351 \mathrm{~kg} / \mathrm{cm}^{2}$ a 28 días y, otro con resistencia de $449 \mathrm{~kg} / \mathrm{cm}^{2}$, según las normas españolas pertenecen a la misma categoría 350 ; mientras que cementos con resistencias de 349 y 351 , ó 449 y $451 \mathrm{~kg} / \mathrm{cm}^{2}$, pertenecen a categorías distintas (250 y 350 , respectivamente, los primeros, y 350 y 450 , respectivamente, los segundos).

Y, a pesar de ello, no cabe ninguna duda acerca de la abismal diferencia entre los cementos 351 y 449 , y la práctica identidad de los cementos 349 y 351, ó 449 y 451 . Este es el 
inconveniente de establecer las categorías de los cementos en las normas por escalones de resistencias mínimas, que pueden dar lugar a exageradas variabilidades dentro de cada categoría.

Por ello sería también recomendable fijar límites, no sólo para las resistencias mínimas a cada edad, sino también para las máximas a la edad de 28 días, dentro de cada categoría. Esta se define entonces por el valor medio del intervalo entre el mínimo y el máximo, si bien según otra alternativa análoga se podría definir también por una resistencia media afectada de una razonable y limitada dispersión.

Así lo han hecho ya algunos países en Europa, y esa parece ser la tendencia. Con ello se procura - $\mathrm{y}$ se consigue- una mayor regularidad de la calidad y una útil y práctica realidad de las categorías de los cementos, con lo cual el constructor y las centrales hormigoneras pueden saber mejor a qué atenerse a la hora de dosificar y elaborar sus hormigones, cuando utilizan en ellos cementos con adiciones.

Esta podría ser una forma de atenuar muy notablemente esa peor parte que, "por ahora", puede parecer que llevaría el usuario en el asunto de los cementos con adiciones, única solución viable a corto plazo para economizar combustible derivado del petróleo en la fabricación de cemento.

Desde el punto de vista de los cementos con adiciones las normas deberían ser más liberales, tendiendo a favorecer, según las circunstancias, el empleo de unas u otras — puzolanas, cenizas, escorias, incluso "fíllers"- en aras, no ya sólo del ahorro de combustibles, sino también de aumentos ide producción, de reducciones de precios, y de eliminación -llámase más bien aprovechamiento- de subproductos, en pro de la ecología y de la descontaminación ambiental.

En determinados países de Europa se ha llegado con éxito a recomendar por parte de la Administración, a determinados estamentos de la misma, el empleo de cementos "con constituyentes secundarios". Así lo tienen en cuenta, además, sus normas oficiales para cemento.

A tales efectos, a los cementos ternarios a base de clínker portland, escoria siderúrgica y puzolana natural o ceniza volante podría estarles reservado un interersante porvenir desde el punto de vista de la durabilidad o resistencia química del hormigón frente a los ataques por agua de mar y por aguas y terrenos yesíferos, o en general sulfatados.

En cuanto a los cementos "fillerizados" - con determinados contenidos de adiciones químicamente inertes (hasta cierto punto), pero con acciones y efectos físicos comprobados-, no son tan desdeñables en determinadas circunstancias, a pesar de sus innegables aspectos deficitarios, pues por su doble o triple capacidad de deformación frente a cargas rápidas, y quíntuple o séxtuple frente a cargas lentas en relación con los cementos ordinarios, pueden ayudar a resolver el problema de la fisuración cuando no existan problemas de fluencia ni se requieran resistencias muy elevadas.

No se quiere dar fin a estos comentarios sin hacer alusión a la promoción del cemento, tanto por vía directa como indirecta, para una aproximación, no ya al ahorro de fuel-oil en la fabricación del material, sino también al ahorro de otros valiosos derivados del petróleo, y por lo tanto de esta problemática materia prima, en campos tan ligados al empleo del cemento como son el de la construcción y el de las obras públicas, y más concretamente las viales. 
Por el lado directo y en el campo de las obras públicas cabe prever en el futuro un empleo masivo y creciente del hormigón, pues las vías de comunicación, los aeropuertos, las obras hidráulicas de todo género, las obras marítimas, las centrales nucleares, etc., con sus respectivas infraestructuras complejas, han de proliferar, y no pueden prescindir deì hormigón como material exclusivo o principal.

El precio del hormigón se prevé en el futuro más competitivo aún que en la actualidad con el de otros materiales, a causa de la escasez, dificultald de adquisición y consiguiente encarecimiento de éstos como consecuencia de la debatida crisis del petróleo y de la energía. En muchos aspectos las ventajas de todo orden, hoy día no totalmente reconoci. das, estarán francamente de parte del hormigón.

En efecto, si escasea el petróleo o se encarece aún más, automáticamente sucederá lo mismo, y tal vez en mayor escala, no sólo con los combustibles, sino también con los plásticos y los productos asfálticos y bituminosos, entre otros.

Y si los derivaldos del petróleo dejan de abundar y suben de precio, se crea una situación en la que la competencia entre el plástico y el amiantocemento, o el fibrocemento en general, se decanta a favor de estos últimos materiales que son consumidores de cemento.

Pero además, por idénticas razones, el petróleo se empleará más como materia prima para la industria química de productos artificiales - entre ellos los plásticos-, que como fuente de combustibles. Y, a su vez, los productos asfálticos y bituminosos, más caros, no se emplearán masivamente en la construcción de nuevos firmes, sino que se reservarán para la reparación y el mantenimiento de los ya construidos y en servicio.

En todo caso, una de las promociones directas más interesantes del empleo del cemento en las obras públicas es la relativa a las pistas, autopistas, carreteras y caminos, es decir, a la vialidad en general. Las mencionadas penuria y carestía de productos bituminosos en un campo tan importante de las obras públicas como es éste de la vialidad puede inclinar la balanza del lado de los firmes blancos y rígidos de hormigón, frente a los firmes negros, flexibles asfálticos, entrando así en vías de estudio y consideración un problema técnico polémico surgido hace años en mi país, y dejado entonces al margen por motivos complejos.

Es este un aspecto en el que la crisis del petróleo se presenta favorable a la promoción del cemento, como ya ha quedado de manifiesto por la elevación de costos de primer establecimiento de firmes negros, experimentada ya y prevista para el futuro en Gran Bretaña, Francia, Bélgica y España, entre otros países, en alguno de los cuales, ante los aumentos de los precios oficiales del asfalto, la Administración ha dado ya hace años instrucciones para que se considere con todo interés la posibilidad de utilizar hormigón en todos los proyectos viarios.

Pero en el aspecto vial no sólo el hormigón constituye un campo de promoción para el cemento, puesto que también lo pueden ser, y en importante medida, otros materiales como la grava-cemento y el suelo-cemento para la estabilización de carreteras secundarias, así como de suelos y terrenos de todo tipo, con cualquier otra finalidad.

En el campo de la construcción, la industrialización de la misma a gran escala parece ser el medio más eficaz y directo para la promoción del cemento. Pero la industrialización integral de la construcción exige el amplio desarrollo de etapas anteriores, como son la mecanización y la automatización, previos a su vez para la prefabricación. 
En definitiva, se trata de construir "en taller" y de montar "en obra", desarrollando la construcción modular y celular (masiva), aprovechando la facilidard que da el hecho de que el hormigón sea un material al que se le ha denominado, un tanto "bárbaramente" (de barbarismo) "formáceo", en vez de "amoldable" —ni siquiera enmoldable o moldeable-. que es lo correcto.

La vivienda social - popular, barata, económica y calificada con tantos otros desafortunados adjetivos, pero en todo caso vivienda masiva desarrollada en serie-, parece ser el más abonado campo de promoción posible del cemento dentro del área de la edificación.

En cuanto a posibles nuevos usos y aplicaciones fuera de la construcción y de las obras públicas, que pudieran contribuir a la promoción del empleo del cemento, se pueden citar, por una parte, y por vía de ejemplo, el de conglomerante para la granulación en frío de menas minerales en polvo, con objeto de hacer rentables procesos siderúrgicos -y metalúrgicos en general- que ahora no lo son con materias primas que hoy no se pueden aprovechar; y, por otra parte, el de coagulante y aglutinante de lodos y barros en suspensión acuosa, como subproductos de procesos industriales, actuando en tal caso como depurador físico de aguas.

De lo primero existe ya el precedente de que con clínker y en determinada forma, se ha logrado aglomerar un polvo de mineral defosforado, difícil de transportar tal cual, y muy caro de granular por sinterización. De lo segundo se sabe que se está trabajando con interés, y parece que con éxito, en un centro europeo de investigación sobre cemento.

En cuanto a las vías indirectas para la promoción del uso del cemento, la formación más especializada de técnicos de fabricación de cemento en cuestiones relativas a la tecnología del hormigón, y de técnicos del hormigón en aspectos de fabricación, normalización y empleo del cemento, con frecuentes contactos mútuos para intercambio de ideas y planteamiento de los respectivos problemas, podría ayudar a ambas partes para conseguir el objetivo común de tratar - cada una en su ámbito- de utilizar los cementos disponibles en cada situación, y particularmente los que ahora y en el futuro puedan contribuir a un ahorro de fuel-oil en la fabricación del material.

La impartición de enseñanzas y conocimientos tecnológicos en tal sentido durante la formación de los técnicos, así como la preparación y difusión de normas, recomendaciones y códigos de buena práctica para el empleo de cementos en hormigones, entre técnicos de la construcción en pleno ejercicio profesional, complementado todo ello con cursos comunes de especialización post-grado para técnicos de ambos campos, cemento y construcción, pueden facilitar a posteriori los mencionados contactos interprofesionales en pro del mayor y mejor empleo del cemento.

El autor ha llegado así al final de su ojeada panorámica a varios de los puntos del temario de esta II Reunión del Grupo Latinoamericano de Instituciones del Cemento y del Concreto, en un intento de relacionarlos entre sí y de exponer, acerca de ellos, su visión personal presente y también un tanto futurista. No le queda, pues, sino agradecer cordialmente la acogida dispensada a lo que pudiera calificarse tal vez de intrusismo, aunque en todo caso lleno de la mejor voluntad.

Por todo ello, y a un mismo tiempo, perdón y gracias. 